TABLE 1. Summary of quantitative and qualitative measures of right ventricular function in patients with pectus excavatum by echocardiography and cardiac magnetic resonance imaging

\begin{tabular}{llccc}
\hline Patient & $\begin{array}{c}\text { RV } \\
\text { dysfunction* }\end{array}$ & $\begin{array}{c}\text { TAPSE } \dagger \\
(\mathbf{c m})\end{array}$ & $\begin{array}{c}\text { RV FAC } \dagger \\
(\%)\end{array}$ & $\begin{array}{c}\text { RV EF } \ddagger \\
(\%)\end{array}$ \\
\hline 1 & Severe & 1.8 & 17.8 & 47 \\
2 & Moderate & 2.5 & 25.5 & 59 \\
3 & Mild & 1.7 & 33.7 & 62 \\
4 & Moderate & 2.2 & 37.0 & 53 \\
5 & Moderate & 1.8 & 41.7 & 50 \\
6 & Moderate & 2.0 & 47.5 & 52 \\
7 & Moderate & 1.0 & 47.7 & 48 \\
8 & Mild & 1.9 & 36.0 & 54 \\
Mean \pm SD & NA & $1.9 \pm 0.5$ & $35.8 \pm 11.2$ & $53.5 \pm 5.4$ \\
Reference range & Normal & $1.5-2.0$ & $32-60$ & $49-72$ \\
$\quad$ in healthy & function & & & \\
subjects $\oint, \|$ & & & & \\
\hline
\end{tabular}

$R V$, Right ventricular; TAPSE, tricuspid annular plane systolic excursion; FAC, fractional area change; $E F$, ejection fraction; $N A$, not applicable. *Qualitative echocardiographic assessment of right ventricular dysfunction as mild, moderate, or severe. $\dagger$ By echocardiography. $\ddagger$ By cardiac magnetic resonance imaging. $§$ Rudski LG, Lai WW, Afilalol J, Hua L, Handschumacher MD, Chandrasekaran K, et al. ${ }^{4} \|$ Saleh RS, Finn JP, Fenchel M, Moghadam AN, Krishnam M, Abrazado M, et al. ${ }^{5}$

An accurate assessment of RV function in patients with pectus excavatum is imperative, because it may alter the decision for corrective surgery. Because of mixed data regarding whether there is measurable cardiopulmonary improvement postoperatively, there is no consensus as to the indications for corrective surgery for pectus excavatum. ${ }^{3}$ Nonetheless, an accurate assessment of biventricular function may help inform the need for surgery and clarify the etiology of symptoms in this anatomically distinct population. Although our case series is limited by a small heterogeneous sample size without a control population, we highlight a potentially important limitation of qualitative echocardiographic assessment when evaluating biventricular function.

\section{References}

1. Kelly RE Jr. Pectus excavatum: historical background, clinical picture, preoperative evaluation, and criteria for operation. Semin Pediatr Surg. 2008;17: 181-93.

2. Mocchegiani R, Badano L, Lestuzzi C, Nicolosi GL, Zanuttini D. Relation of right ventricular morphology and function in pectus excavatum to the severity of the chest wall deformity. Am J Cardiol. 1995;76:941-6.

3. Malek MH, Berger DE, Housh TJ, Marelich WD, Coburn JW, Beck TW. Cardiovascular function following surgical repair of pectus excavatum: a meta- analysis. Chest. 2006;130:506-16.

4. Rudski LG, Lai WW, Afilalo J, Hua L, Handschumacher MD, Chandrasekaran K, et al. Guidelines for the echocardiographic assessment of the right heart in adults: a report from the American Society of Echocardiography endorsed by the European Association of Echocardiography, a registered branch of the European Society of Cardiology, and the Canadian Society of Echocardiography. J Am Soc Echocardiogr. 2010;23:685-713; quiz, 786-8.

5. Saleh RS, Finn JP, Fenchel M, Moghadam AN, Krishnam M, Abrazado M, et al. Cardiovascular magnetic resonance in patients with pectus excavatum compared with normal controls. J Cardiovasc Magn Reson [serial online]. Available from: http://jcmr-online.com/content/pdf/1532-429X-12-73.pdf. December 13, 2010;12:73. Accessed August 1, 2011.

\title{
A new echocardiographic window to visualize the mitral valve complex during mitral valve repair for functional mitral regurgitation
}

\author{
Kikuko Obase, MD, PhD, ${ }^{\mathrm{a}}$ Masashi Komeda, $\mathrm{MD}, \mathrm{PhD},{ }^{\mathrm{b}}$ Hiroyuki Okura, $\mathrm{MD}, \mathrm{PhD},{ }^{\mathrm{a}}$ and \\ Kiyoshi Yoshida, MD, PhD, ${ }^{\text {a }}$ Kurashiki and Nagoya, Japan
}

Reconstructive surgery, which restores the normal alignment between the mitral annulus and displaced papillary muscles (PMs), might be beneficial in patients with functional mitral regurgitation (MR). A detailed understanding

From the Department of Cardiology, ${ }^{\text {a }}$ Kawasaki Medical School, Kurashiki, Japan; and Department of Cardiovascular Surgery, ${ }^{\mathrm{b}}$ Nagoya Heart Center, Nagoya, Japan Disclosures: Authors have nothing to disclose with regard to commercial support.

Received for publication Nov 14, 2011; revisions received Jan 11, 2012; accepted for publication Jan 24, 2012; available ahead of print Feb 20, 2012.

Address for reprints: Kikuko Obase, MD, PhD, Department of Cardiology, Kawasaki Medical School, 577 Matsushima, Kurashiki 701-0192, Japan (E-mail: obasekkk@ med.kawasaki-m.ac.jp).

J Thorac Cardiovasc Surg 2012;143:e42-4

$0022-5223 / \$ 36.00$

Copyright (C) 2012 by The American Association for Thoracic Surgery

doi:10.1016/j.jtcvs.2012.01.064 of the mitral apparatus is the key to successful surgery. Although 3-dimensional transesophageal echocardiography (3D-TEE) plays an essential role in understanding the mitral valve apparatus, the conventional trans-left atrial (LA) observation by 3D-TEE sometimes fails to visualize the subvalvular apparatus. When assisting surgical subvalvular reconstruction, it is imperative to obtain appropriate images for quantitative analysis of the submitral morphology. In the present study, we compared the effectiveness of the transgastric approach using 3D-TEE in visualizing the mitral valve structure with the conventional trans-LA approach during surgery for functional MR.

\section{METHODS}

A total of 8 consecutive patients ( 2 with chronic atrial fibrillation) underwent mitral valve surgery for functional MR. After anesthesia induction 


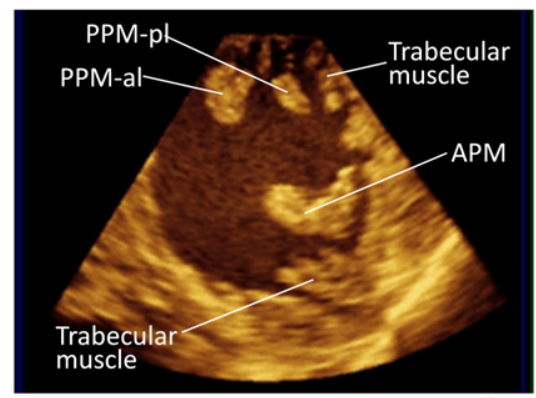

A
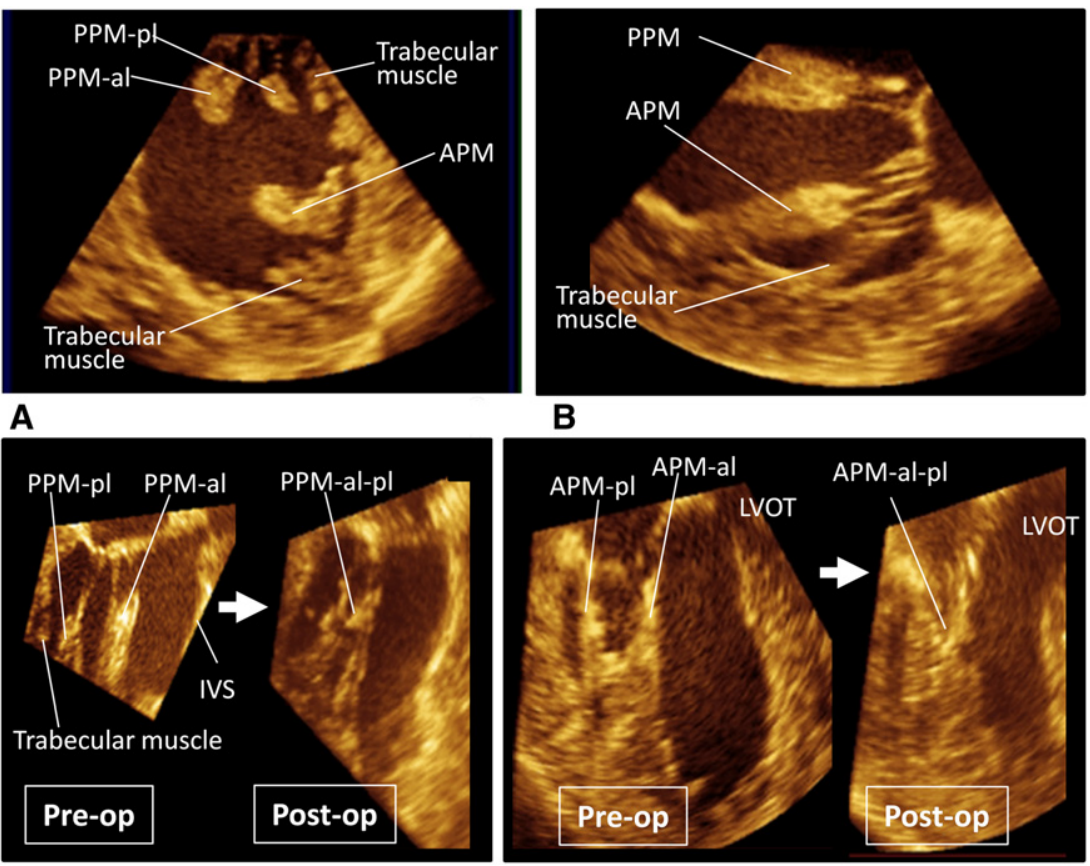

B

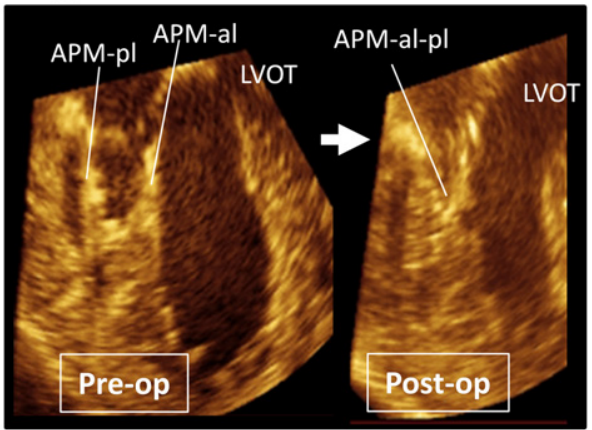

C

D

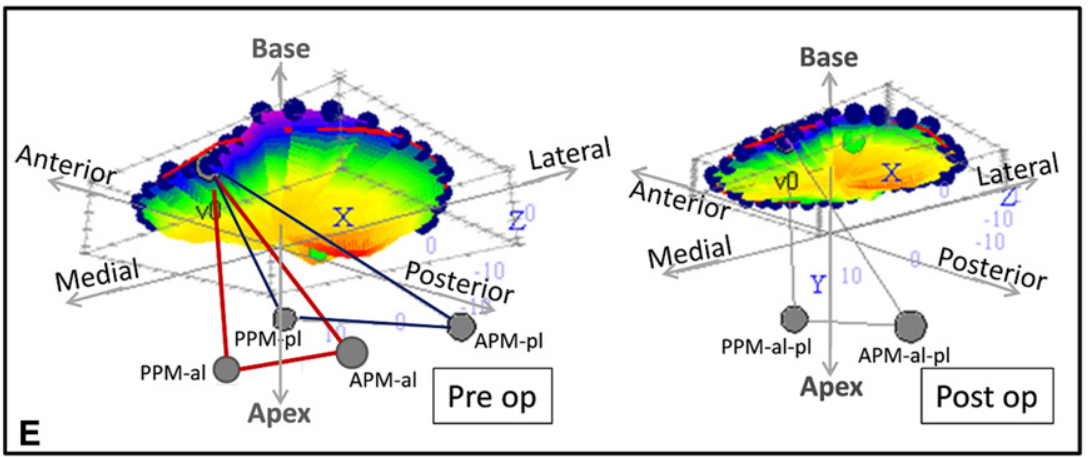

FIGURE 1. Visualizing submitral structure at midsystole from 3-dimensional data set acquired by transgastric approach using QLAB, with short-axis view (A), coronal section (commissure-commissure direction) view (B), sagittal section (anteroposterior leaflet direction) view of posterior papillary muscle $(P P M)(\mathrm{C})$, anterior papillary muscle $(A P M)(\mathrm{D})$, and 3-dimensional reconstructed annulus, leaflets, and position of papillary muscle $(P M)$ heads for quantitative analysis using Real View (E). PPM-al and APM-al, PPM and APM head for anterior leaflet, respectively; PPM-pl and APM-pl, PPM and APM head for posterior leaflet, respectively; $L V O T$, left ventricular out tract. Operatively connected PM head for posterior leaflet and anterior leaflet was clearly visualized as PPM-al-pl and APM-al-pl, respectively.

and endotracheal intubation, full-volume 3D-TEE data were acquired using both the trans-LA and the transgastric approaches, pre- and postoperatively. After surgically exposing the mitral valve, the number of PMs, the heads in each PM, and the chordal distribution were observed and recorded verbally by the surgeon. All patients underwent a novel surgical procedure, developed from the chordal translocation method, ${ }^{1}$ as follows. The PM head for the posterior leaflet was connected to the PM head for the anterior leaflet using a polytetrafluoroethylene suture in each PM. Next, its arms were resuspended toward the mid-anterior mitral annulus. The anatomy of the PM was interpreted from the full volume 3D-TEE data using the multiplanar reformatting mode of QLAB (Philips Medical Systems, Andover, Mass; Figure 1, $A-D$ ) on cart and compared with the surgeon's dictation. Off-line quantitative analysis was performed using Real View (YD, Nara, Japan; Figure 1,E). All patients gave informed consent before surgery. The institutional review board of Nagoya's Heart Center approved the study protocol.

\section{RESULTS}

In all patients, the interpretation of PM division and chordal distribution by transgastric TEE corresponded exactly with the intraoperative dictation by the surgeon. The transgastric approach was superior to the trans-LA approach in visualizing the heads of the PM and chordae tendineae distribution for quantitative analysis, with $75 \%$ and $87.5 \%$ satisfactory and $25 \%$ and $12.5 \%$ acceptable results, respectively. In visualizing the leaflets and annulus, the trans-LA approach was superior to the transgastric approach (Table 1). A typical case is shown in Figure 1. The PM heads in each PM were clearly visualized. With the aid of quantitative software, these images allowed accurate 
TABLE 1. Number of patients stratified by visualizing feasibility

\begin{tabular}{|c|c|c|c|c|c|c|c|c|}
\hline \multirow[b]{2}{*}{ Visualization } & \multicolumn{4}{|c|}{ Trans-LA } & \multicolumn{4}{|c|}{ Transgastric } \\
\hline & Leaflet & Annulus & PM division & Chordal distribution & Leaflet & Annulus & PM division & Chordal distribution \\
\hline Satisfactory & $6(75)$ & $6(75)$ & 0 & 0 & $3(37.5)$ & $4(50)$ & $6(75)$ & $7(87.5)$ \\
\hline Acceptable & $2(25)$ & $2(25)$ & $1(12.5)$ & 0 & $4(50)$ & $4(50)$ & $2(25)$ & $1(12.5)$ \\
\hline Inadequate & 0 & 0 & 7 (87.5) & $8(100)$ & $1(12.5)$ & 0 & 0 & 0 \\
\hline
\end{tabular}

Data presented as numbers, with percentages in parentheses. Trans- $L A$, Trans-left atrial; $P M$, papillary head.

measurement of the pre- and postoperative inter-PM head distance, the distance between the PM head and midanterior annulus and the tenting volume, and so forth (Figure 1,E).

\section{DISCUSSION}

The pre- and postoperative study of the PMs and chordae tendineae is essential in ascertaining the most suitable mitral valve repair strategy for MR of different etiologies and also for evaluating the results of valve repair. Adequate imaging is necessary for precise quantitative evaluation of the mitral valve structure. The trans-LA approach provides an almost perpendicular echo beam to the leaflets. It is therefore an ideal approach to visualize the leaflets during systole. However, the trans-LA echo beam is parallel to the chordae tendineae and PMs and attenuates before it reaches the PM, resulting in suboptimal images of the subvalvular apparatus. The transgastric echo beam is from the direction of the inferior wall by way of the gastric wall and diaphragm and is perpendicular to the chordae tendineae and PMs, allowing clear visualization of the submitral structure. The transgastric approach is, therefore, not suitable for visualizing leaflets in systole.

\section{CONCLUSIONS}

The transgastric approach offers complementary information to the trans-LA approach for the quantitative evaluation of the mitral valve complex. The transgastric approach should be added to the conventional approach as a new echocardiographic window during mitral repair for functional MR.

\section{Reference}

1. Masuyama S, Marui A, Shimamoto T, Komeda M. Chordal translocation: secondary chordal cutting in conjunction with artificial chordae for preserving valvularventricular interaction in the treatment of functional mitral regurgitation. J Heart Valve Dis. 2009;18:142-6. 